Noman 2015, 33(1), 57-68

Revista de Psicologia, Ciències de l'Educació i de l'Esport

ISSN: $1138-3194$

Copyright (C) 2015

www.revistaaloma.net

\title{
Perfil Clínico de Apego (PCA): elaboración de un sistema de categorías para la evaluación del apego
}

\author{
Glòria Lucena, Ignacio Cifre, José A. Castillo-Garayoa \& Elena Aragonés \\ Universitat Ramon Llull
}

Recibido: 29-10-2014

Aceptado: 27-3-2015

Perfil Clínico de Apego (PCA): elaboración de un sistema de categorías para la evaluación del apego

Resumen. A partir de la teoría de Bowlby y de los estilos de apego propuestos por Bartholomew y Horowitz, se desarrolla el Perfil Clínico de Apego-narrativas (PCA-n), un sistema de observación para evaluar el apego a partir de las narrativas de los y las pacientes. En el Estudio 1, se construye una primera versión del instrumento (PCAv1). La consistencia interna resulta adecuada en la evaluación del apego seguro y evitativo, pero insuficiente en el caso del apego preocupado y temeroso. En el Estudio 2 se introducen cambios en el instrumento (PCA-n), que finalmente consta de cuatro categorías: disponibilidad-confianza, autonomía en la relación, regulación de las emociones y revelación de las emociones. Aplicado a las narrativas de dos pacientes, se obtiene un nivel de acuerdo entre evaluadores en la identificación de las narrativas significativas superior al 75\%. También resulta adecuada la fiabilidad del PCA-n, ya que la correlación promedio en la identificación de las categorías fue .78 en el caso del paciente 1, y .88 en el paciente 2.Se discuten las aplicaciones posibles del PCA-n, sus ventajas y limitaciones, así como líneas de investigación futuras.

Palabras clave: apego, evaluación observacional, narrativas, psicopatología, psicoterapia

\section{Clinical Attachment Profile (CAP): creation of a system of categories to assess attachment}

Summary. This article makes use of Bowlby's theory and the attachment styles set out by Bartholomew and Horowitz, in order to to develop an Clinical Attachment Profile-narratives (CAP-n), an observation system for the assessment of attachment using patient narratives. In Study 1, an initial version of this tool is built (ACPv1). The tool displays sufficient levels of internal consistency in the assessment of both secure and avoidant attachment, but not in that of anxious and fearful attachment styles. In Study 2 changes are made to the instrument (CAP-n), which finally features four categories: availability-trust, autonomy in the relationship, regulation of emotions and communication of emotions. When the model is applied to the narratives of two patients, the degree of inter-assessor agreement in the identification of significant narratives exceeds $75 \%$. The reliability of the CAP-n is also sufficient in that the average correlation in the identification of the categories was .78 for one patient and .88 for the other. The article discusses possible applications of the CAP-n, its advantages and limitations and possibilities for further research.

Keywords: attachment, observational assessment, narratives, psychopathology, psychotherapy

Correspondencia:

José A. Castillo-Garayoa

C/Císter, 34

Teléfono: 932533000

Dirección correo electrónico: JosepAntonicc@blanquerna.url.edu 


\section{Introducción}

La teoría del apego constituye un marco fundamental para el estudio de las relaciones interpersonales. Para Bowlby (1986), los humanos nacemos con la predisposición biológica a vincularnos, a buscar proximidad, protección y seguridad en otros humanos. Esa búsqueda de proximidad se activa ante la percepción de una amenaza, cuando sentimos ansiedad o miedo. En la definición clásica de Ainsworth y Bowlby (1991), se denomina apego al vínculo emocional que se establece entre el niño o la niña y uno o más cuidadores por los que muestra preferencia, con los que se siente seguro/a, receptivo/a a las manifestaciones de afecto, y de los que teme separarse. Un apego seguro se desarrolla a partir de que las figuras de apego respondan de forma sensible y apropiada a las necesidades físico-emocionales del niño; el apego ambivalente o de resistencia sería consecuencia de una respuesta inconsistente, unas veces adecuada y otras no, mientras que el apego evitativo sería el resultado de un vínculo caracterizado por el abandono, el rechazo y la frialdad.

A partir de los primeros vínculos con las figuras de apego se van acumulando experiencias relacionales que, sobre todo en la adolescencia, configuran modelos operativos internos, es decir, esquemas cognitivoemocionales, expectativas y atribuciones de significado que se activan de manera automática y que configuran patrones de relación con los demás. Los modelos operativos internos reflejan el predominio de una imagen positiva o negativa de uno mismo (self) y de los demás (Bowlby, 1988; Marrone, 2008; Melero \& Cantero, 2008; Soares \& Dias, 2007). Este es el punto de partida de Bartholomew (Bartholomew, 1990; Bartholomew \& Horowitz, 1991) para sus cuatro tipos de apego adulto: seguro, evitativo, preocupado y temeroso.

Como puede observarse en la Figura 1, en el apego seguro, el modelo mental tanto de uno mismo como del otro es positivo. Este tipo de apego corresponde a personas con adecuada autoestima y confianza en sí mismas y en los demás, y que se consideran dignas de atención y de ayuda. Valoran la intimidad, se sienten cómodas en ella y pueden mantener el equilibrio entre las necesidades afectivas y la autonomía personal.

El apego evitativo se caracteriza por el predominio de una imagen positiva de uno mismo y negativa del otro, de manera que la prioridad fundamental es mantener la creencia de que no se necesita a nadie. Para ello, las personas con apego evitativo tienden a desactivar las necesidades de apego y a evitar la intimidad. El sentimiento de autosuficiencia está exacerbado porque desconfían y quitan valor a los demás. En el apego preocupado la imagen de uno mismo es negativa y la de los demás positiva, de manera que hay carencia de autoestima y una elevada activación del sistema de apego; suelen ser personas dependientes, que necesitan la aprobación de los demás y temen el abandono. De ahí que nos refiramos a confianza «ciega» en los demás: la gran necesidad de vincularse puede reducir la capacidad de observar al otro, de conocerlo, de percibir sus posibles defectos. La persona de apego temeroso, por último, tiene un modelo mental negativo tanto de sí mismo como de los demás. Son personas que necesitan el contacto interpersonal, pero tienden a evitarlo por temor al rechazo y al abandono. Se sienten incómodas en situaciones de intimidad, aunque necesitan la aprobación para mantener su autoestima alta (Bartholomew \& Horowitz, 1991; Melero \& Cantero, 2008; Yárnoz, 2008; Yárnoz, AlonsoArbiol, Plazaola \& Sainz de Murieta, 2001).

Los cuatro modelos de apego corresponden también a distintas combinaciones de dos dimensiones básicas del apego: la ansiedad, que se genera en las relaciones interpersonales, y la evitación, la preferencia por una mayor o menor distancia con los demás. La proximidad e intimidad en las relaciones promueven más ansiedad en las personas de apego preocupado y temeroso, y menos en las de apego seguro y evitativo. Respecto a la evitación, esta es mayor en las personas de apego evitativo y temeroso, que prefieren mantener distancia interpersonal y evitar la intimidad (Yárnoz, 2008).

\begin{tabular}{|c|c|c|c|}
\hline & & \multicolumn{2}{|c|}{ (-) ------- Evitación ------- (+) } \\
\hline & & $\begin{array}{c}\text { Expectativa sobre los demás } \\
\text { Positiva }\end{array}$ & $\begin{array}{c}\text { Expectativa sobre los demás } \\
\text { Negativa }\end{array}$ \\
\hline 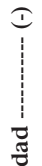 & $\begin{array}{l}\text { Autoconcepto } \\
\text { Positivo }\end{array}$ & $\begin{array}{c}\text { Seguro } \\
\text { Comodidad con la intimidad, autonomía, facilidad para } \\
\text { las relaciones interpersonales } \\
\text { Confianza básica }\end{array}$ & $\begin{array}{c}\text { Evitativo } \\
\text { Desactivación del sistema de apego, se evitan las } \\
\text { relaciones íntimas } \\
\text { Desconfianza (distante) }\end{array}$ \\
\hline$\stackrel{Ð}{ \pm}$ & $\begin{array}{l}\text { Autoconcepto } \\
\text { Negativo }\end{array}$ & $\begin{array}{c}\text { Preocupado } \\
\text { Dependencia, deseo de aprobación, miedo al abandono } \\
\text { Confianza «ciega» }\end{array}$ & $\begin{array}{c}\text { Temeroso } \\
\text { Miedo al rechazo, experiencia de frustración en las } \\
\text { relaciones } \\
\text { Desconfianza (temerosa) }\end{array}$ \\
\hline
\end{tabular}

Figura 1. Estilos de apego adulto a partir de los modelos del self y de los demás.

Fuente: Bartholomew, K. (1990). Avoidance of intimacy: An attachment perspective. Journal of Social and Personal Relationships, 7, 147-178; Bartholomew, K. \& Horowitz, L. (1991). «Attachment styles among young adults: a test of a four-category model». Journal of Personality and Social Psychology, 61(2), 226-244.

Nota fuente: adaptado de Castillo, J.A. \& Medina, P. (2007). Maltrato en la relación de pareja: apego, intimidad y cambios sociales. En A. Talarn (Comp.), Globalización y salud mental (pp. 393-416). Barcelona: Herder. 
Además de las aportaciones de los modelos de apego adulto para la comprensión de las relaciones interpersonales en contextos familiares, sociales y de pareja, resulta de gran utilidad evaluar el apego en la práctica clínica. Centrándonos en el trabajo con pacientes adultos, conviene tener en cuenta la relación entre apego, psicopatología y psicoterapia. Así, existe abundante investigación que asocia los apegos inseguros (temeroso, preocupado, evitativo) a mayor presencia de sintomatología ansiosa y depresiva, dificultades de relación y de adaptación y, en general, a un mayor riesgo de desarrollar trastornos psicopatológicos (Allen, Porter, McFarland, McElhaney \& Marsh, 2007; Camps, Castillo \& Cifre, 2014; Fonagy et al., 1996; Keskin \& Çam, 2010; McLewin \& Muller, 2006; Muller, Lemieux \& Sicoli, 2001; Soares \& Dias, 2007).

Respecto a la psicoterapia, el tipo de apego del paciente es una variable fundamental para el proceso y el resultado del tratamiento (Eagle, 2006; Lilliengren et al., 2014; McBride, Atkinson, Quilty \& Powell, 2006). Con mayor o menor énfasis, todas las orientaciones psicoterapéuticas coinciden en la importancia de la alianza terapéutica, uno de cuyos componentes es el vínculo emocional que se establece entre paciente y terapeuta. Al igual que, como subraya la teoría del apego, todo humano necesita vínculos personalizados e íntimos para lograr un desarrollo adecuado, en la psicoterapia esa necesidad será también un requisito imprescindible para el cambio (Fonagy, 2004). El tipo de apego del paciente y del terapeuta serán determinantes para facilitar o entorpecer la formación de la alianza terapéutica y para entender cómo ambos afrontan lo que hay de íntimo en su relación (Bernecker, Levy \& Ellison, 2014; Beutler, Castonguay \& Follette, 2006; Lossa, Corbella \& Botella, 2012). En las primeras entrevistas se planteará la oportunidad de establecer un nivel adecuado de empatía, proximidad y confianza, la base de una sólida alianza terapéutica, o bien aparecerán dificultades debido a la intensa necesidad de distancia interpersonal, a la suspicacia, al miedo al rechazo, la dependencia o la frialdad. Sin embargo, el tipo de apego no es inmodificable: se ha desarrollado a partir de experiencias relacionales y el cambio se producirá también en el contexto de la relación que se establezca en terapia. Dicha relación ha de tener elementos nuevos, ha de ser diferente a la vivida por el paciente, y ha de fomentar la función reflexiva, es decir, la capacidad para entender la mente del otro y la propia (Fonagy \& Allison, 2014; Fonagy, Steele, Moran, Steele \& Higgitt, 1991; Gullestad, Johansen, Hoglend, Karterud \& Wilberg, 2013).

Por lo comentado hasta ahora, es útil para la práctica clínica disponer de instrumentos que permitan evaluar el apego de los pacientes. La Entrevista del Apego Adulto (Adult Attachment Interview, AAI) de George, Caplan y Main (1985) es el instrumento de evaluación del apego adulto más sofisticado y utilizado para la investigación. Consiste en una entrevista semiestructurada de-mínimo- una hora de duración que determina el apego a partir de las narraciones que la persona hace sobre sus relaciones con las figuras de apego. Además de determinar el apego del paciente, la AAI permite guiar la intervención terapéutica y evaluar su resultado (Steele, Steele \& Murphy, 2009; Yárnoz et al., 2001; Yárnoz \& Comino, 2011).

Centrándose en el apego que se establece en la relación de pareja, Hazan y Shaver (1987) desarrollaron el Cuestionario de estilo de apego adulto, un autoinforme en el que se pide a la persona que escoja una de las tres descripciones que se le presentan, cada una de ellas refleja un estilo relacional prototípico y característico del apego seguro, ambivalente y evitativo (Yárnoz, 2008).

En España, Melero y Cantero (2008) han desarrollado el Cuestionario de apego adulto, un autoinforme de cuarenta ítems que permite evaluar cuatro tipos de apego (seguro, preocupado, temeroso-hostil y alejado). También disponemos de versiones adaptadas de otros instrumentos de autoinforme, como el CaMir (Balluerka, Lacasa, Gorostiaga, Muela \& Pierrehumbert, 2011), el ECR-R (Fernández-Fuertes, Orgaz, Fuertes \& Carcedo, 2011) o el EPERIC (Fontanil, Ezama \& Alonso, 2013).

Destacar finalmente el Cuestionario de Relación-CR (Relationship Questionnaire, RQ) de Bartholomew y Horowitz (1991), utilizado en el presente estudio. El CR es un autoinforme que presenta cuatro descripciones sobre formas de relacionarse; se pide a la persona evaluada que elija aquella con la que se sienta más identificada y, a continuación, que puntúe cada una de las cuatro descripciones en una escala de siete puntos. Cada descripción corresponde a uno de los cuatro estilos de apego propuestos por Bartholomew: seguro, preocupado, evitativo y temeroso. El mismo cuestionario permite evaluar también las dimensiones ansiedad y evitación, calculadas a partir de las puntuaciones en cada uno de los subtipos de apego (Bartholomew \& Horowitz, 1991; Yárnoz, 2008; Yárnoz \& Comino, 2011). Resultados recientes de la aplicación del CR refieren dos dimensiones subyacentes diferentes a ansiedad y evitación, en concreto inseguridad del apego (baja en el apego seguro y alta en el temeroso) y estrategias para afrontar esa inseguridad (busca de proximidad en el apego preocupado y distancia interpersonal en el apego evitativo) (Sheinbaum, Bedoya, Kwapil \& BarrantesVidal, 2013).

A pesar de contar con instrumentos diversos, como los referidos, la evaluación del apego en contextos clínicos continúa presentando dificultades. Así, la Entrevista de apego adulto AAI se utiliza principalmente para la investigación, ya que implica introducir en el proceso de evaluación psicológica clínica una entrevista semiestructurada específica (cosa que no siempre es fácil), y su administración y corrección exigen una acreditación profesional que se consigue después de una formación y un entrenamiento complejo y de difícil acceso (Steele \& Steele, 2003 en Yárnoz, 2008; Yárnoz et al., 2001). Por otro lado, los cuestionarios de autoinforme (Cuestionario de apego adulto, CR, CaMir, ECR-R, EPERIC) se basan en la perspectiva del paciente, en los que pueden verse los resultados alterados por 
sus sesgos perceptivos, por las limitaciones de su autoconocimiento o por la deseabilidad social.

Es por estos motivos que se ha creado un instrumento para la evaluación del apego adulto en contextos clínicos, un instrumento que no implique modificar el proceso de evaluación clínica que prefiera seguir cada profesional, que no comporte una formación larga y/o compleja y que evite los sesgos propios de una medida de autoinforme. El Perfil Clínico de Apegonarrativas (PCA-n) puede aplicarse a las narrativas de los pacientes sobre sus relaciones con personas significativas (padre, madre, hermanos o hermanas, pareja, etc.) y consigo mismos. A continuación se presentará el trabajo realizado hasta el momento, subdivido en dos partes: en el Estudio 1 se diseña la primera versión del Perfil Clínico de Apego (PCAv1), mientras que en el Estudio 2 se optimiza esa primera versión, lo que da como resultado el PCA-n, y se forma a un grupo de evaluadores que lo administran.

\section{Estudio 1}

\section{Método}

En el Estudio 1 se construye la primera versión del Perfil Clínico del Apego (PCAv1) y se aplica a narrativas de cuatro pacientes, y se contrastan los resultados del PCAv1 con la autoevaluación del apego realizada por dichos pacientes a través del Cuestionario de Relación (CR) de Bartholomew y Horowitz (1991).

\section{Participantes}

Se analizan las narrativas realizadas por cuatro pacientes jóvenes en los respectivos procesos de evaluación psicológica clínica. Los jóvenes, tres chicas y un chico, de edades comprendidas entre 17 y 19 años, han sufrido maltrato intrafamiliar (negligencia -cuatro pacientes-, maltrato físico y psicológico -tres pacientes-, abusos sexuales -un paciente-) y residen en pisos asistidos bajo la supervisión de un equipo educativo. Presentan problemáticas psicológicas diversas (somatizaciones, ansiedad, depresión, dificultades de relación, etc.), han solicitado ayuda por iniciativa propia o por indicación de algún referente institucional, y son atendidos en un servicio concertado de la red pública de servicios sociales. En la primera entrevista, los pacientes firman el consentimiento informado que autoriza el uso de sus datos para fines de investigación. Del conjunto de jóvenes atendidos en el servicio, los cuatro de la muestra se han seleccionado a partir de un muestreo no probabilístico de tipo intencional.

La transcripción de las entrevistas anamnésicas de los cuatro pacientes (véase apartado «Procedimiento»), una vez eliminada la información para garantizar el anonimato, constituyó el material clínico al que se aplicó el PCAv1.

\section{Instrumentos}

Perfil Clínico de Apego-versión 1 (PCAv1). Se ha construido la primera versión del Perfil Clínico de Apego-versión
1 (PCAv1), un sistema de categorías para evaluar el estilo de apego a partir de las narrativas que el paciente hace sobre sí mismo y sobre los demás en el curso de entrevistas clínicas o sesiones de psicoterapia. El PCAv1 se enmarca en el modelo de apego adulto de Bartholomew, que distingue cuatro tipos de apego (seguro, preocupado, temeroso y evitativo) en función de la percepción que la persona tiene de sí mismo (self) y del otro. Las categorías observacionales del PCAv1 se han planteado a partir de conceptos y temas centrales en la teoría del apego, teniendo en cuenta las aportaciones de autores relevantes (Bartholomew \& Horowitz, 1991; Bowlby, 1986; Cassidy \& Shaver, 2008; Hazan \& Shaver, 1987), así como las revisiones de Melero y Cantero (2008) y Yárnoz (2008). El PCAv1 incluye las categorías y subcategorías siguientes: 1. Seguridad (confianza, fiabilidad, autorrevelación); 2. Disponibilidad (accesibilidad, cuidado, autonomía, relación de poder); 3. Experimentación de las emociones y relevancia de la relación (regulación de las emociones, ansiedad); y 4. Imagen (positiva y negativa). Cada una de esas categorías podía referirse al propio paciente (self) o a personas significativas de su entorno (otros), y tener un valor positivo o negativo. Partiendo de transcripciones de entrevistas de evaluación y/o de tratamiento en que se recogen las narrativas del paciente, el procedimiento de aplicación del PCAv1 es el siguiente (una explicación más detallada puede leerse en el Estudio 2 y en el Anexo 1):

1. Seleccionar las unidades de análisis, es decir, aquellas narrativas en que el paciente se refiere a un aspecto del self o de otros: padre, madre, familia, pareja, etc.

2. Determinar a qué categoría/s corresponde cada unidad de análisis.

3. Contabilizar la frecuencia total de narrativas clasificadas en cada categoría.

4. Sumar los valores de cada categoría y contabilizar la puntuación correspondiente a cada tipo de apego, así como a las dimensiones ansiedad y evitación vistas en la Figura 1.

\section{Cuestionario de Relación (CR)}

El Cuestionario de Relación (CR) es un autoinforme breve que fue desarrollado por Bartholomew y Horowitz (1991) para evaluar el estilo de apego de personas adultas a partir de medidas continuadas y resultados categóricos. En primer lugar, se presentan a la persona evaluada cuatro descripciones prototípicas de los tipos de apego del modelo de Bartholomew (seguro, preocupado, evitativo y temeroso), y se le pide que decida con cuál se identifica más. En segundo lugar, se le pide que puntúe el grado de acuerdo con cada una de las definiciones prototípicas de apego, en una escala Likert de siete puntos (Yárnoz, 2008; Yárnoz, et al., 2001). Los cuatro pacientes de la muestra cumplimentaron el CR en el marco del proceso de evaluación clínica que realizaron.

\section{Procedimiento y análisis de datos}

El material clínico de los cuatro pacientes fue evaluado por tres observadores, conocedores del modelo de 
Bartholomew y de las aplicaciones clínicas de la teoría del apego. Dos de las evaluadoras son psicólogas con estudios de posgrado en psicología de la salud y psicoterapia, y el tercer evaluador es el psicólogo clínico que atendió a los jóvenes cuyas narrativas se utilizan para el estudio.

Los tres evaluadores aplicaron el protocolo de uso del PCA a las narrativas de los cuatro pacientes. Se seleccionaron las narrativas correspondientes a la entrevista anamnésica; una entrevista semiestructurada en la que se explora la infancia del paciente (embarazo y parto, alimentación, marcha, habla, control de esfínteres, relaciones familiares), su escolaridad y formación académica (rendimiento académico, relación con docentes y compañeros), el ámbito laboral, sus relaciones de amistad y de pareja, y posibles problemas de salud, incluyendo consumo de sustancias. Una parte de la anamnesis consiste en pedir al paciente que describa a personas significativas para él, y que se autodescriba.

Los cuatro pacientes de la muestra habían cumplimentado el Cuestionario de Relación (CR) como parte del proceso de evaluación psicológica clínica que se realizó, de manera que pudo contrastarse la evaluación del apego obtenida a partir del análisis de las narrativas de los pacientes (PCA) con la referida por ellos mismos a partir de un autoinforme (CR).

\section{Resultados}

\section{Acuerdo entre observadores}

En primer lugar se ha calculado el acuerdo bruto entre parejas de evaluadores (entre el evaluador 1 y el 2, entre el 1 y el 3, y entre el 2 y el 3) en las narrativas de los cuatro pacientes, es decir, el número de veces que clasifican una misma narrativa en una misma categoría. El acuerdo bruto entre observadores se ha transformado en porcentajes, calculados como el cociente entre «acuerdos» $\mathrm{y}$ «acuerdos + desacuerdos». Como aparece reflejado en la Tabla 1, el acuerdo entre el observador 1 y el 2 oscila entre el 42.59\% (paciente 4) y el $74.07 \%$ (paciente 3 ). Entre el evaluador $1 \mathrm{y}$ el 3, las concordancias oscilan entre el 37 y el 63\%, mientras que las observaciones de los evaluadores 2 y 3 coinciden en porcentajes que van del 40.7 al 63\%. Por tanto, los observadores 1 y 2 muestran mayor concordancia en sus observaciones, en comparación al observador 3. Es en la evaluación de las narrativas del paciente 4 donde el acuerdo entre observadores tiende a ser menor.
Tabla 2. Tipos de apego predominante de los pacientes, desde la perspectiva del PCAv1 y del autoinforme CR.

\begin{tabular}{lll}
\hline & PCAv1 & CR \\
\hline Paciente 1 & $\begin{array}{l}\text { Preocupado } \\
\text { Temeroso }\end{array}$ & $\begin{array}{l}\text { Seguro } \\
\text { Temeroso }\end{array}$ \\
\hline Paciente 2 & $\begin{array}{l}\text { Evitativo } \\
\text { Preocupado }\end{array}$ & $\begin{array}{l}\text { Evitativo } \\
\text { Seguro }\end{array}$ \\
\hline Paciente 3 & Temeroso & $\begin{array}{l}\text { Preocupado } \\
\text { Evitativo }\end{array}$ \\
\hline Paciente 4 & Temeroso & Evitativo \\
& Evitativo & Temeroso \\
\hline
\end{tabular}

Nota: En negrita se indica el tipo de apego predominante, y en cursiva el segundo.

\section{Consistencia interna}

La consistencia interna del PCAv1 fue evaluada a partir del coeficiente intraclase de Berk $\left(p^{2}\right)$, que calcula la fiabilidad indicando la concordancia absoluta entre las medidas de los tres observadores para los distintos tipos de apego (seguro, evitativo, preocupado y temeroso). Este coeficiente de concordancia global tiene la característica de ser sensible a las posibles sobreestimaciones por parte de algún observador. El índice $p^{2}{ }_{c}$ del estilo de apego seguro $\left(p^{2}{ }_{c}=.72\right)$ y evitativo $\left(p^{2}{ }_{c}=.76\right)$ obtienen una fiabilidad alta como categorías. Por contra, la concordancia en la observación del apego preocupado (.44) y temeroso (.54) no alcanza suficiente fiabilidad.

\section{Tipo de apego de los pacientes según el PCAv1 y el CR}

En la Tabla 2 se recogen los estilos de apego predominantes de cada paciente, considerando la perspectiva conjunta de los tres evaluadores que aplicaron el PCAv1, y la perspectiva del propio paciente, autoevaluada mediante el CR. La autoevaluación realizada por el paciente 4 es la que se aproxima más a la realizada mediante el PCAv1 por los observadores, ya que desde ambas perspectivas predominan los estilos evitativo y temeroso, si bien en orden inverso. Por contra, en los pacientes 1, 2 y 3, la perspectiva de los pacientes y del PCAv1 es coincidente en uno de los dos estilos de apego (paciente 1: temeroso; paciente 2: evitativo; paciente 3: evitativo) pero divergen en la evaluación del segundo estilo de apego predominante.

\section{Estudio 2}

A partir de los resultados obtenidos en el Estudio 1, se optimiza el PCAv1 y se forma a un grupo de evaluadores para su administración, con el objetivo de calcular la fiabilidad del instrumento y comprobar su aplicabilidad por parte de evaluadores no expertos.

Tabla 1. Acuerdo bruto entre los observadores 1, 2 y 3, en la categorización de las narrativas de los cuatro pacientes de la muestra.

\begin{tabular}{lccc}
\hline & Observadores 1-2 & Observadores 1-3 & Observadores 2-3 \\
\hline Paciente 1 & $26(48.15 \%)$ & $24(44.4 \%)$ & $25(46.3 \%)$ \\
\hline Paciente 2 & $27(50 \%)$ & $24(44.4 \%)$ & $22(40.7 \%)$ \\
\hline Paciente 3 & $40(74.07 \%)$ & $34(63 \%)$ & $34(63 \%)$ \\
\hline Paciente 4 & $23(42.59 \%)$ & $20(37 \%)$ & $23(42.6 \%)$ \\
\hline
\end{tabular}




\section{Método}

\section{Participantes}

La nueva versión del Perfil Clínico de Apego-narrativas (en adelante, PCA-n) se ha aplicado a las narrativas de dos pacientes, un chico y una chica de 18 y 19 años, respectivamente, que han sufrido maltrato intrafamiliar y consultan a un servicio de atención psicológica (véanse más detalles en el Estudio 1). Aplicaron el PCA$\mathrm{n}$ un total de 54 evaluadores, 2 de los autores del instrumento (en adelante, evaluadores-expertos) y 52 estudiantes (evaluadores-no-expertos) de tercer curso del Grado de Psicología de la Facultat de Psicologia Blanquerna (URL), alumnos de la asignatura Evaluación y Psicopatología en Adultos.

\section{Instrumentos}

Teniendo en cuenta las deficiencias observadas en el PCAv1, se ha construido la segunda versión del Perfil Clínico de Apego, PCA-n (Anexo 1), un sistema de categorías para evaluar el estilo de apego a partir de las narrativas que el paciente hace sobre sí mismo y sobre los demás en el curso de entrevistas clínicas o sesiones de psicoterapia (véase en el Estudio 1 el proceso de construcción del instrumento y su fundamento teórico).

Las categorías del PCA-n (Anexo 1) están englobadas en cuatro grupos: 1. Disponibilidad-confianza (presenciaausencia en uno mismo y en los demás de seguridad, confianza y fiabilidad), 2. Autonomía en la relación (autonomía adecuada, apoyarse en exceso por temor al abandono, no apoyarse por temor a sufrir, negación de la necesidad de relacionarse), 3. Regulación de las emociones (regulación adecuada, hiperactivación de las emociones, desactivación de las emociones), y 4. Revelación de las emociones (autorrevelación adecuada, autorrevelación excesiva-indiscriminada, dificultad para autorrevelarse). Todas las categorías se aplican a la visión que el paciente tiene de sí mismo, y las categorías de disponibilidad-confianza se aplican también a la visión del paciente sobre los demás. Cada categoría del PCA-n puntúa de manera positiva o negativa en ansiedad y en evitación, las dos dimensiones subyacentes al modelo de apego de Bartholomew, de manera que la aplicación del instrumento permite determinar si el tipo de apego del paciente es seguro (ansiedad y evitación negativas), preocupado (ansiedad positiva, evitación negativa), temeroso (ansiedad positiva y evitación positiva) o evitativo (ansiedad negativa y evitación positiva).

Partiendo de transcripciones de entrevistas de evaluación y/o de tratamiento en que se recogen las narrativas del paciente, el procedimiento de aplicación del PCA-n es el siguiente:

1. Seleccionar las unidades de análisis, es decir, aquellas narrativas en que el paciente se refiere a un aspecto del self o de personas o grupos de personas significativas para él (otros): padre, madre, familia, iguales, pareja, general (referencia a «la gente», «todo el mundo», etc.).
2. Determinar a qué categoría/s corresponde cada unidad de análisis. En una unidad de análisis puede haber información relevante para más de una categoría.

3. Contabilizar la frecuencia total de narrativas clasificadas en cada categoría y convertir las frecuencias en valores de probabilidad, calculando el cociente entre la frecuencia de cada categoría y el número total de observaciones. Mediante la conversión en valores de probabilidad se busca poder comparar a pacientes en el tipo de verbalizaciones que realizan sobre sus relaciones de apego, con independencia de que dichas verbalizaciones sean muchas o pocas.

4. Sumar los valores de cada categoría y contabilizar la puntuación correspondiente a cada tipo de apego. Tal y como veíamos en la Figura 1, las valoraciones positivas del self corresponderían, en el modelo de Bartholomew, al apego seguro y al evitativo, mientras que las valoraciones negativas computarían en los apegos preocupado y temeroso. Las valoraciones positivas que el paciente hace de los demás computarían en los estilos de apego seguro y preocupado, mientras que las valoraciones negativas de los demás computarían en los apegos evitativo y temeroso.

\section{Procedimiento y análisis de datos}

Dos de los autores del PCA-n (evaluadores-expertos) aplicaron el instrumento a las narrativas de los dos pacientes a los que se hacía referencia anteriormente, y se consensuaron las divergencias hasta llegar a un resultado unívoco. A continuación, se realizó una sesión formativa sobre la aplicación del PCA-n, en la que participaron estudiantes de tercer curso del Grado de Psicología (véase apartado de «Participantes»). En dicha sesión formativa se recordaba a los estudiantes el fundamento teórico del instrumento, el modelo de apego adulto de Bartholomew (ya tratado con anterioridad en la asignatura), y se explicaba el procedimiento de aplicación del instrumento.

Después de la sesión formativa, 24 evaluadores-noexpertos aplicaron el PCA-n a la narrativa de uno de los pacientes, mientras que 28 lo aplicaron a la narrativa del segundo paciente. Las narrativas estaban separadas por párrafos temáticos, en cada uno de los cuales el paciente se refería a un tema, experiencia, opinión, etc. Se pidió a los evaluadores que marcasen con una $\mathrm{X}$ los párrafos donde el paciente refiriera algún aspecto relacionado con la visión de sí mismo y/o de los demás. En aquellos párrafos marcados con una X, debían codificar cada una de las categorías del sistema.

\section{Resultados}

Como paso previo al cálculo de la fiabilidad del sistema, debíamos conocer si los evaluadores-no-expertos eran capaces de identificar las frases con contenido relevante para la evaluación del apego. Para ello, se calculó la precisión (grado en que la valoración del evaluadorno-experto coincide con la valoración del evaluadorexperto) en términos de sensibilidad y especificidad de 
cada evaluador-no-experto respecto al evaluador-experto. La sensibilidad es el grado en que el evaluadorno-experto es capaz de identificar una frase como significativa para la evaluación del apego, cuando el evaluador-experto así lo ha establecido. La especificidad es el grado en que el evaluador-no-experto es capaz de identificar una frase como «no significativa», coincidiendo con la valoración del evaluador-experto. La sensibilidad de los evaluadores-no-expertos de las narrativas del paciente 1 (21 frases significativas, de un total de 27) fue $\mathrm{x}=.75$ ( $\mathrm{dt}=.09$ ) y la de los evaluadoresno-expertos del paciente 2 , con 26 frases significativas de un total de 31 , fue $\mathrm{x}=.64(\mathrm{dt}=.12)$. Respecto a la especificidad, en el paciente 1 fue $\mathrm{x}=.80(\mathrm{dt}=.22) \mathrm{y}$ en el paciente $2, \mathrm{x}=.92(\mathrm{dt}=.12)$.

Se calculó el porcentaje para cada una de las 58 frases (narrativas de los pacientes) analizadas, es decir, el porcentaje frase-a-frase en que los evaluadores-noexpertos coincidían con los evaluadores-expertos. El resultado mostró que en 47 frases, el porcentaje de acuerdo era superior a $75 \%$.

Centrándonos ya en las categorías del PCA-n, a continuación se calculó la correlación de Spearman para cada uno de los evaluadores-no-expertos con el evaluador-experto, es decir, si coincidían las categorías atribuidas a cada narrativa por parte de los evaluadoresno-expertos con las atribuidas por el evaluador-experto (grado de concordancia global). Los resultados muestran que, en el paciente 1, el promedio de correlaciones de los evaluadores-no-expertos con el evaluador-experto fue $.78(\mathrm{dt}=.07)$. De las 28 correlaciones referidas al paciente 1,6 resultan estadísticamente significativas a nivel $\mathrm{p}<.05$ y 22 a nivel $\mathrm{p}<.001$. En el paciente 2 , el promedio de correlaciones de los evaluadores-no-expertos con el evaluador-experto fue .88 $(\mathrm{dt}=.09)$. De las 24 correlaciones referidas al paciente 2,1 resulta estadísticamente significativas a nivel $\mathrm{p}<.05$ y 23 a nivel $\mathrm{p}<.001$.

Dado que el cálculo de la correlación no informa sobre la infraestimación o la sobreestimación de un observador, se calculó el coeficiente intraclase de Berk. Considerando, en primer lugar, el conjunto de categorías del sistema, sin diferenciar por tipo de apego, el coeficiente de Berk es $p_{c}^{2}=.77$ para el paciente 1 y $p_{c}^{2}=.88$ para el paciente 2. Considerando los diferentes tipos de apego, el coeficiente de Berk para las categorías asociadas al apego seguro fueron $p_{c}^{2}=.74$ (paciente 1) y $p^{2}{ }_{c}=.79$ (paciente 2); para el apego evitativo $p_{c}^{2}=.93$ (paciente 1) y $p_{c}^{2}=.87$ (paciente 2); para el apego preocupado, $p^{2}=.53$ (paciente 1 ) y $p_{c}^{2}=.52$ (paciente 2); para el apego temeroso, $p_{c}^{2}=.78$ (paciente 1$)$ y $p_{c}^{2}=.82$ (paciente 2).

Una vez calculada la fiabilidad, se ha calculado el resultado de aplicar el PCA-n a cada uno de los pacientes en términos de ansiedad y evitación. Recordemos que cada categoría del PCA puntúa positiva o negativamente sobre una o ambas dimensiones. En la figura 2 se representan las puntuaciones de evitación y de ansiedad que resultan de las evaluaciones realizadas por los evaluadores-no-expertos (rombos oscuros) y los evaluadores-expertos (rombo claro). En ambos casos, la mayoría de las evaluaciones se situaría alrededor de la recta, lo que indica que dichas evaluaciones están relacionadas. La mayoría de las evaluaciones del paciente 1 realizadas mediante el PCA se sitúan en el cuadrante ansiedad baja y evitación alta, correspondiente al apego evitativo; en la administración del CR, el paciente 1 también autopercibía su apego como evitativo. En el caso del paciente 2, las puntuaciones confluirían en el cuadrante ansiedad alta y evitación alta, correspondiente al apego temeroso, en tanto que el paciente se autopercibe como preocupado.

\section{Discusión y conclusiones}

En el presente estudio se construye un sistema de categorías para evaluar, en contextos clínicos, las representaciones cognitivo-emocionales sobre uno mismo (self) y los demás que constituyen los modelos operativos internos (Bowlby, 1988). El Perfil Clínico de Apegonarrativas (PCA-n) se basa en el modelo de apego adulto de Bartholomew (Bartholomew, 1990; Bartholomew \& Horowitz, 1991) y se aplica a las narrativas de los pacientes referidas tanto a sí mismos como a los demás (padres, iguales, pareja, etc.). Además de la correlación existente entre apego y psicopatología (Allen et al., 2007; Camps, Castillo \& Cifre, 2014; Fonagy et al., 1996; Keskin \& Çam, 2010; McLewin \& Muller,

Figura 2. Gráfico de dispersión de las evaluaciones realizadas por el evaluador-experto (rombo claro) y por los evaluadores-noexpertos (rombos oscuros), del paciente 1 (gráfico de la izquierda) y del paciente 2 (gráfico de la derecha).
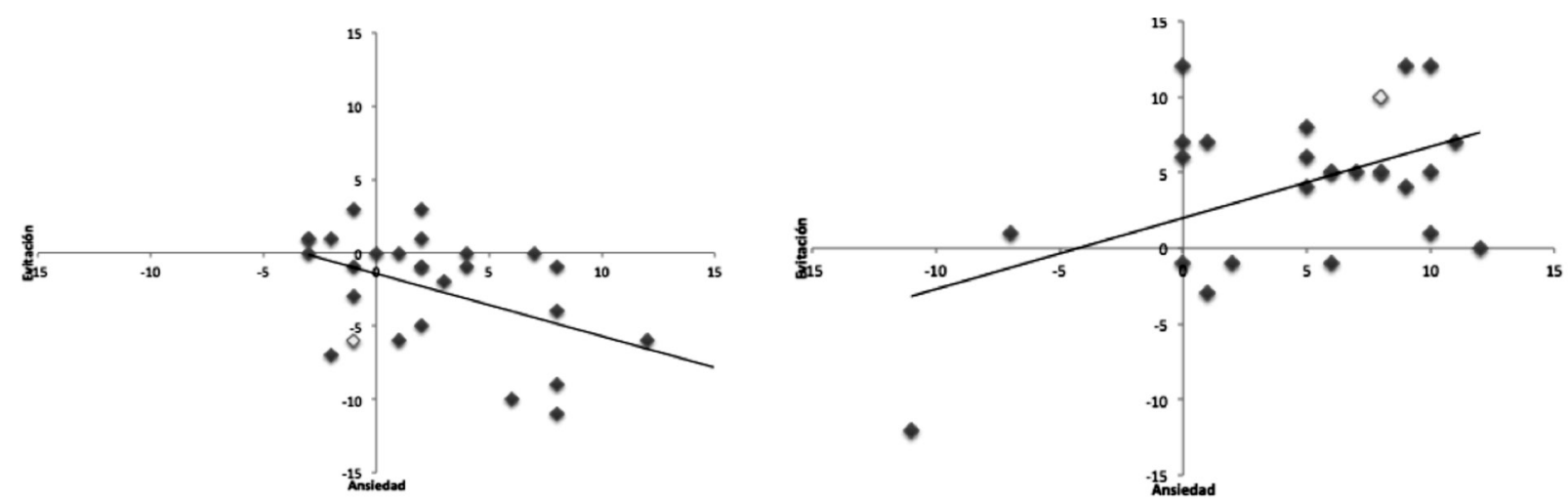
2006; Muller, Lemieux \& Sicoli, 2001; Soares \& Dias, 2007), evaluar el estilo de apego permite comprender mejor la dinámica relacional del paciente, los patrones relacionales que tienden a repetirse en sus relaciones, sus expectativas y atribuciones de significado, así como su forma de entender y afrontar la intimidad con los demás. Todo ello facilita la empatía del profesional hacia el paciente, el establecimiento de una alianza terapéutica más sólida y el trabajo terapéutico (Bernecker, Levy \& Ellison, 2014; Beutler, Castonguay \& Follette, 2006; Lossa, Corbella \& Botella, 2012).

En la primera parte de este trabajo (Estudio 1) se ha construido una primera versión del Perfil clínico de apego (PCAv1). Tres evaluadores aplicaron el instrumento a las narrativas de cuatro pacientes, y compararon el tipo de apego resultante de la aplicación del PCAv1 con los resultados del Cuestionario de Relación (CR) de Bartholomew y Horowitz (1991), autoinforme con el que el paciente evalúa su estilo de apego desde su propia perspectiva.

El análisis psicométrico del PCAv1 ha mostrado una adecuada fiabilidad en las categorías correspondientes al apego seguro y al evitativo, si bien no era suficiente la fiabilidad alcanzada por los observadores al evaluar las categorías de apego preocupado y temeroso. La causa de la falta de fiabilidad se halló en algunas dificultades de definición de las categorías y en la tendencia a sobreestimar ocurrencias de conducta por parte de uno de los evaluadores. De los tres evaluadores que participaron en el estudio, dos habían trabajado en la construcción del instrumento, y fue el tercero -menos familiarizado con el procedimiento de aplicaciónquien tendía a considerar que en la narrativa de los pacientes había más referencias a temas relacionados con el apego de las que codificaban los otros evaluadores.

Un resultado interesante del Estudio 1, a nuestro juicio, es que aparecen discrepancias entre la evaluación del apego realizada por los pacientes mediante el CR y la evaluación del apego realizada mediante el PCA. El sentido de trabajar un nuevo instrumento de evaluación del apego radica precisamente en la posibilidad de observar esas discrepancias, que -como veremos- se confirman en el Estudio 2. A partir de ahí se revisó el instrumento, se redujo el número de categorías (de 27 a 12) y se clarificó su definición. Las categorías finales del PCA-n (Anexo 1) se engloban en cuatro grupos: disponibilidad-confianza (presencia-ausencia en uno mismo y en los demás de seguridad, confianza y fiabilidad), autonomía en la relación (autonomía adecuada, apoyarse en exceso por temor al abandono, no apoyarse por temor a sufrir, negación de la necesidad de relacionarse), regulación de las emociones (regulación adecuada, hiperactivación de las emociones, desactivación de las emociones) y revelación de las emociones (autorrevelación adecuada, autorrevelación excesiva-indiscriminada, dificultad para autorrevelarse). Esas categorías recogen las principales variables de la teoría del apego: proximidad, seguridad, confianza, autonomía, autoestima, cuidado y protección, disponibilidad, compro- miso, intimidad, evitación y regulación de las emociones (Bartholomew \& Horowitz, 1991; Bowlby, 1986; Cassidy \& Shaver, 2008; Hazan \& Shaver, 1987; Melero \& Cantero, 2008; y Yárnoz, 2008).

En el Estudio 2 se aplica el PCA-n a la narrativa de dos jóvenes adultos, y se comparan las valoraciones realizadas por evaluadores expertos (dos de los autores del instrumento) con las de un grupo de evaluadores no expertos, estudiantes del Grado de Psicología. Los resultados indican que el PCA-n es un instrumento fácil de aplicar, incluso por evaluadores en periodo de formación, sin conocimientos clínicos y teóricos profundos. Los evaluadores-no-expertos localizaron adecuadamente las unidades de análisis, aquellos fragmentos de la narrativa del paciente que son significativos para evaluar el apego, y además categorizaron dichas unidades de análisis de manera concordante con la evaluación realizada por los expertos. Esta facilidad para aprender a utilizar el PCA-n, con una formación clínica limitada y en un tiempo breve, resulta prometedora de cara a la diseminación del instrumento en contextos clínicos y de investigación. Se abre, pues, una nueva vía de evaluación del apego, compatible con los autoinformes y con instrumentos tan sofisticados como la entrevista de apego adulto (AAI).

Tanto en el Estudio 1 como en el Estudio 2 se observan diferencias entre los tipos de apego resultantes de la aplicación del PCA-n y los tipos de apego autoinformados por los propios pacientes a través del CR. Este es uno de los aspectos clave del trabajo, que deberá confirmarse en el futuro con más muestras. Por el momento, no podemos saber qué fuente de información (el propio paciente o la observación de sus narrativas) es más fiable y útil para la práctica clínica. Conviene tener presente que los pacientes de la muestra han sufrido maltrato, y que existe suficiente evidencia sobre el efecto del maltrato en la capacidad de mentalización (Fonagy, 2004; Fonagy \& Allison, 2014). Parece plausible que el paciente tenga dificultades para autopercibirse de una manera realista y libre de sesgos. El desarrollo de la función reflexiva o capacidad de mentalización requiere del predominio de una respuesta sensible por parte de las figuras de apego, de un ambiente familiar que fomente el pensamiento y la reflexión sobre uno mismo y los demás, y los padres y madres de los jóvenes de la muestra están ausentes, o sufren adicciones y trastornos mentales graves, así como otros problemas psicosociales (maltrato en la relación de pareja, enfermedades, delincuencia, prostitución) que no son una base segura para el desarrollo psicoemocional y relacional.

La evaluación psicológica clínica y la intervención con jóvenes que han sufrido maltrato deben tener presentes las aportaciones sobre la relación entre apego y psicoterapia, y contemplar además que la incidencia de apegos inseguros será alta. La inseguridad en el apego, la hiperactivación o desactivación de las emociones, las dificultades para la regulación de la distancia relacional y para establecer un vínculo de confianza afectan directamente a una de las piezas centrales 
del trabajo clínico: la solidez de la alianza terapéutica (Bernecker, Levy \& Ellison, 2014; Beutler, Castonguay \& Follette, 2006; Lossa, Corbella \& Botella, 2012). El paciente de apego evitativo tenderá a mostrarse invulnerable, seguro de sí mismo, evitará el estrés y la frustración poniendo barreras al reconocimiento de dificultades, a la expresión de las mismas y a la recepción de ayuda, incluida la profesional. Una de las pacientes de la muestra expresaba así el freno a la autorrevelación personal, su voluntad de desactivar experiencias dolorosas y de sentirse invulnerable al dolor que pueden suscitar algunas relaciones: «me abro poco a los demás», «tengo muchas cosas que echarle en cara, pero me da igual» (refiriéndose a su padre). En el caso de las personas de apego preocupado, Yárnoz (2008) explica que la exageración en la manifestación de sus necesidades de apego aumenta las posibilidades de ser atendidos. Los jóvenes con estilo preocupado se muestran vulnerables y frágiles, y esta actitud les permite muchas veces acceder a la respuesta esperada: la atención y el cuidado del otro.

En este contexto, es imprescindible tener en cuenta la peculiaridad relacional del paciente, como acabamos de ver, y la interacción que habrá entre el estilo de apego del paciente con el del profesional. Un profesional de apego seguro tiene más facilidad para mostrarse flexible en su relación con el paciente y para tolerar los conflictos que puedan aparecer. Un profesional de apego preocupado, con cierto miedo al rechazo, puede sentirse muy afectado por la actitud de frialdad y autosuficiencia de un paciente con apego evitativo. Un profesional de apego evitativo puede ser percibido como rechazante por un paciente temeroso, muy sensible a esa experiencia relacional. Y así debería examinarse cada díada, para anticipar dificultades y ajustar el tipo de relación más adecuado (Lilliengren et al., 2014; Mallinckrodt, 2000; Marmarosh et al., 2014). El paciente planteará la relación terapéutica a partir de sus modelos operativos internos, que le sitúan en un escenario relacional previsible y, por tanto, menos ansiógeno. Uno de los problemas es que el profesional confirme esos modelos, se ajuste a las expectativas del paciente en lugar de cuestionarlas, sobreinterviniendo con pacientes hiperactivadores de las emociones (preocupados), y guardando más distancia de la debida con pacientes desactivadores (evitativos y temerosos).

El Perfil Clínico de Apego (PCA-n) contempla tanto la perspectiva categorial como dimensional del apego. El sistema permite evaluar la presencia de diferentes estrategias relacionales, propias de diferentes tipos de apego, y también clasificar el estilo de apego predominante de un paciente. La perspectiva dimensional parece más útil, si bien esa misma perspectiva admite que un tipo de apego destaca sobremanera sobre el resto. En cualquier caso, debemos evitar posturas reduccionistas y deterministas. El estilo de apego puede modificarse a partir de un trabajo personal y/o psicoterapéutico. El estilo de apego puede adaptarse, evolucionar, cambiar, y sobre todo puede pensarse: reflexionar sobre los modelos operativos internos propios y desarrollar la mentalización serían objetivos de cualquier psicoterapia (Allen \& Fonagy, 2006).

El PCA-n es una primera aportación a la evaluación de las representaciones sobre uno mismo y los demás asociadas al apego. Para ello utiliza un sistema de categorías que debe continuar desarrollándose en el futuro. Además de seguir trabajando en su fiabilidad y consistencia, un aspecto importante a considerar sería la incorporación al instrumento de características cualitativas del discurso del paciente. Un desarrollo en esta línea puede ser contemplar la calidad y la coherencia del discurso, su emocionalidad, la riqueza de las evidencias aportadas por el paciente para apoyar sus comentarios sobre sí mismo y/o los demás. Aumentar el número de casos evaluados, así como las tipologías de pacientes, es también un trabajo imprescindible. El PCA-n puede ser un instrumento que facilite la evaluación del apego en contextos clínicos, a partir de lo que el paciente explica, sin alterar la práctica habitual del profesional.

\section{Referencias}

Ainsworth, M.D.S. \& Bowlby, J. (1991). An ethological approach to personality development. American Psychologist, 46, 331-341. doi:10.1037//0003066X.46.4.333

Allen, J.G. \& Fonagy, P. (2006). Handbook of mentalization-based treatment. New York: Wiley. doi:10.1002//9780470712986

Allen, J.P., Porter, M., McFarland, C., McElhaney, K.B. \& Marsh, P. (2007). The relation of attachment security to adolescent's paternal and peer relationships, depression, and externalizing behavior. Child Development, 78(4), 1222-1239. doi:10.1111/j.14678624.2004.00817.x

Balluerka, N., Lacasa, F., Gorostiaga, A., Muela, A. \& Pierrehumbert, B. (2011). Versión reducida del cuestionario CaMir (CaMir-R) para la evaluación del apego. Psicothema, 23(3), 486-494. doi:21774904

Bartholomew, K. (1990). Avoidance of intimacy: An attachment perspective. Journal of Social and Personal Relationships, 7, 147-178. doi:10.1177/ 0265407590072001

Bartholomew, K. \& Horowitz, L. (1991). Attachment styles among young adults: a test of a four-category model. Journal of Personality and Social Psychology, 61(2), 226-244. doi:10.1037//0022-3514.61.2.226

Bernecker, S.L., Levy, K.N. \& Ellison, W.D. (2014). A meta-analysis of the relation between patient adult attachment style and the working alliance. Psychotherapy Research, 24(1), 12-24. doi:10.1080/10503307.2 013.809561

Beutler, L.E., Castonguay, L.G. \& Follette, W.C. (2006). Therapeutic factors in dysphoric disorders. Journal of Clinical Psychology, 62(6), 639-647. doi:10.1002/ jclp. 20260

Bowlby, J. (1986). Vínculos afectivos: formación, desarrollo y pérdida. Madrid: Morata. 
Bowlby, J. (1988). Una base segura: aplicaciones clínicas de una teoría del apego. Barcelona: Paidós.

Camps, S., Castillo, J.A. \& Cifre, I. (2014). Apego y psicopatología en adolescentes y jóvenes que han sufrido maltrato: implicaciones clínicas. Clínica y Salud, 25(1), 67-74. doi:10.5093/cl2014a6

Cassidy, J. \& Shaver, P.R. (Eds.) (2008). Handbook of attachment: theory, research, and clinical applications. New York: Guilford.

Castillo, J.A. \& Medina, P. (2007). Maltrato en la relación de pareja: apego, intimidad y cambios sociales. En A. Talarn (Comp.), Globalización y salud mental (pp. 393-416). Barcelona: Herder.

Eagle, M.N. (2006). Attachment, psychotherapy, and assessment: A commentary. Journal of Consulting and Clinical Psychology, 74(6), 1086-1097. doi:10.1037/0022-006X.74.6.1086

Fernández-Fuertes, A.A., Orgaz, B., Fuertes, A. \& Carcedo, R. (2011). La evaluación del apego romántico en adolescentes españoles: validación de la versión reducida del Experiences in Close Relationships-Revised (ECR-R). Anales de Psicología, 27(3), 827-833. doi:10.6018/135561

Fonagy, P. (2004). Teoría del apego y psicoanálisis. Barcelona: Espaxs.

Fonagy, P. \& Allison, E. (2014). The role of mentalizing and epistemic trust in the therapeutic relationship. Psychotherapy, 51(3), 372-380. doi:10.1037/a0036505

Fonagy, P., Leigh, T., Steele, M., Steele, H., Kennedy, R., Mattoon, G., et al. (1996). The relation of attachment status, psychiatric classification, and response to psychotherapy. Journal of Consulting and Clinical Psychology, 64, 22-31. doi:10.1037//0022-006X. 64.1 .22

Fonagy, P., Steele, H., Moran, G., Steele, M. \& Higgitt, A. (1991). The capacity for understanding mental states: The reflective self in parent and child and its significance for security of attachment. Infant Mental Health Journal, 13, 200-217. doi:10.1002/10970355(199123)12:3\%3C201::AID-IMHJ2280120307\% 3E3.0.CO;2-7

Fontanil, Y., Ezama, E. \& Alonso, Y. (2013). Validation of the Scale of Preferences and Expectations in Close Interpersonal Relationships (EPERIC). Psicothema, 25(2), 275-281. doi: 10.7334/psicothema2012.125

George, C., Caplan, N. \& Main, M. (1985). Attachment Interview for Adults. Unpublished manuscript. Berkeley: University of California.

Gullestad, F.S., Johansen, M.S., Hoglend, P., Karterud, S. \& Wilberg, T. (2013). Mentalization as a moderator of treatment effects: Findings from a randomized clinical trial for personality disorders. Psychotherapy Research, 23(6), 674-689. doi:10.1080/10503307.201 2.684103

Hazan, C. \& Shaver, P. (1987). Conceptualizing romantic love as an attachment process. Journal of Personality and Social Psychology, 52(3), 511-524. doi:10.1037// 0022-3514.52.3.511

Keskin G. \& Çam, O. (2010). Adolescent's strenghts and difficulties: approach to attachment styles. Jour- nal of Psychiatric and Mental Health Nursing, 17, 433441. doi:10.1111/j.1365-2850.2009.01540.x

Lilliengren, P., Werbart, A., Mothander, P.R., Ekström, A., Sjögren, S. \& Ögren, M.-L. (2014). Patient Attachment to Therapist Rating Scale: Development and psychometric properties. Psychotherapy Research, 24(2), 184-201. doi:10.1080/10503307.2013.867462

Lossa, L., Corbella, S. \& Botella L. (2012). Estilo de apego del paciente en la construcción de la alianza terapéutica. Boletín de Psicología, 105, 91-103.

Mallinckrodt, B. (2000). Attachment, social competencies, social support, and interpersonal process in psychotherapy. Psychotherapy Research, 10(3), 239266. doi:10.1093/ptr/10.3.239

Marmarosh, C.L., Kivlighan, D.M., Bieri, K., Schutt, J.M.L., Barone, C. \& Choi, J. (2014). The insecure psychotherapy base: Using client and therapist attachment styles to understand the early alliance. Psychotherapy, 51(3), 404-412. doi:10.1037/a0031989

Marrone, M. (2008). La teoría del apego como paradigma psicoanalítico. En S. Yárnoz, La Teoría del Apego en la Clínica, I (p.15-36). Madrid: Psimática.

McBride, C., Atkinson, L., Quilty, L.C. \& Powell, B. (2006). Attachment as moderator of treatment outcome in major depression: A randomized control trial of interpersonal psychotherapy versus cognitive behavior therapy. Journal of Consulting and Clinical Psychology, 74(6), 1041-1054. doi:10.1037/0022006X.74.6.1041

McLewin, L. A. \& Muller, R. T. (2006). Attachment and social support in the prediction of psychopathology among young adults with and without a history of physical maltreatment. Child Abuse \& Neglect, 30, 171-191. doi:10.1016/j.chiabu.2005.10.004

Melero, R. \& Cantero, M.J. (2008). Los estilos afectivos en la población española: un cuestionario de evaluación del apego adulto. Clínica y salud, 19(1), 83-100.

Muller, R.T., Lemieux, K.E. \& Sicoli, L.A. (2001). Attachment and Psychopathology among formerly maltreated adults. Journal of Family Violence, 16(2), 151-168. doi:10.1023/A:1011158919303

Sheinbaum, T., Bedoya, E., Kwapil, T.R. \& BarrantesVidal, N. (2013). Comparison of self-reported attachment in young adults from Spain and the United States. Psicothema, 25(4), 514-519. doi:10.7334/psicothema2012.345

Soares, I. \& Dias, P. (2007). Apego y psicopatología en jóvenes y adultos: contribuciones recientes de la investigación. International Journal of Clinical and Health Psychology, 7(1), 177-195.

Steele, H., Steele, M. \& Murphy, A. (2009). Use of the Adult Attachment Interview to measure process and change in psychotherapy. Psychotherapy Research, 19(6), 633-643. doi:10.1080/10503300802609698

Steele, H., \& Steele, M. (2003). Clinical uses of the Adult Attachment Interview. In M. Cortina, \& M. Marrone (Eds.), Attachment Theory and the Psychoanalytic Process (pp. 107-126). London: Whurr.

Yárnoz, S. (2008). La Teoría del Apego en la Clínica, I: 
Evaluación y clínica. Madrid: Psimática.

Yárnoz, S., Alonso-Arbiol, I., Plazaola, M. \& Sainz de Murieta, L.M. (2001). Apego en adultos y percepción de los otros. Anales de psicología, 17(2), 159170.

Yárnoz, S. \& Comino, P. (2011). Evaluación del apego adulto: Análisis de la convergencia entre diferentes instrumentos. Acción Psicológica, 8(2), 67-85. doi:10.5944/ap.8.2.191

\section{Perfil Clínic d'Afferament (PCA): elaboració d'un sistema de categories per a l'avaluació de l'aferrament}

Resum. A partir de la teoria de Bowlby i dels estils d'aferrament proposats per Bartholomew i Horowitz, es desenvolupa el Perfil Clínic d'Aferrament-narratives (PCA-n), un sistema

ANEXO 1: PERFIL CLÍNICO DE APEGO-narrativas (PCA-n): sistema de categorías y procedimiento de aplicación. (Puede solicitarse a los autores una descripción más completa de las categorías.)

Partiendo de transcripciones de entrevistas de evaluación y/o de tratamiento en que se recogen las narrativas del paciente, el procedimiento de aplicación del PCA-n es el siguiente:

1. Seleccionar las unidades de análisis, es decir, aquellas narrativas en que el paciente se refiere a un aspecto d'observació per avaluar l'aferrament a partir de les narratives dels pacients. En l'estudi 1, es construeix una primera versió de l'instrument (PCAv1). La consistència interna resulta adequada en l'avaluació de l'afferament segur i evitatiu, però insuficient en el cas de l'aferrament preocupat i temorós. A l'estudi 2 s'introdueixen canvis en l'instrument (PCA-n), que finalment consta de quatre categories: disponibilitat-confiança, autonomia en la relació, regulació de les emocions i revelació de les emocions. Aplicat a les narratives de dos pacients, s'obté un nivell d'acord entre avaluadors en la identificació de les narratives significatives superior al 75\%. També resulta adequada la fiabilitat del PCA-n, ja que la correlació mitjana en la identificació de les categories va ser .78 en el cas del pacient 1 , $i 88$ en el pacient 2. Es discuteixen les aplicacions possibles del $P C A-n$, les seves avantatges i limitacions, així com línies d'investigació futures.

Paraules clau: aferrament, avaluació observacional, narratives, psicopatologia, psicoteràpia de sí mismo (self) o de personas o grupos de personas significativas para él (otros): padre, madre, familia, iguales, pareja, general (referencia a «la gente», «todo el mundo», etc.).

2. Determinar a qué categoría/s corresponde cada unidad de análisis: si el paciente hace referencia a la confianza-disponibilidad que tiene/percibe en sí mismo y/o en los demás (categorías 1.1. y 1.2), a la autonomía en la relación (categorías 2.1., 2.2., 2.3., 2.4.), la regulación de las emociones (3.1., 3.2., 3.3.) y/o la

\begin{tabular}{|c|c|c|c|c|}
\hline \multicolumn{5}{|c|}{ Categorías del PERFIL CLÍNICO DE APEGO-narrativas (PCA-n) } \\
\hline & Self ${ }^{1}$ & Otros $^{1}$ & Ansiedad $^{2}$ & Evitación $^{2}$ \\
\hline \multicolumn{5}{|l|}{ 1. DISPONIBILIDAD-CONFIANZA } \\
\hline $\begin{array}{l}\text { 1.1. Está disponible cuando se le requiere. Confía, está seguro. Se considera fiable él } \\
\text { mismo y/o los demás. Reconoce virtudes, capacidades. }\end{array}$ & & & - & - \\
\hline $\begin{array}{l}\text { 1.2. No está disponible cuando se le requiere. No confía, no está seguro. No se } \\
\text { considera fiable a sí mismo/a y/o a los demás. Reconoce defectos, dificultades y } \\
\text { problemas. }\end{array}$ & & & + & + \\
\hline 2. AUTONOMÍA EN LA RELACIÓN & \multicolumn{2}{|l|}{ Self $^{1}$} & & \\
\hline $\begin{array}{l}\text { 2.1. Es autónomo/a. Valora y confía en la capacidad propia para establecer una } \\
\text { relación. }\end{array}$ & & & - & - \\
\hline 2.2. Falta autonomía, se apoya en exceso por temor al abandono. Se deja dominar. & & & + & - \\
\hline $\begin{array}{l}\text { 2.3. Falta autonomía pero no se apoya en los demás. Le gustaría hacerlo pero teme } \\
\text { sufrir. }\end{array}$ & & & + & - \\
\hline $\begin{array}{l}\text { 2.4. Busca autonomía negando su necesidad de los demás. No se apoya en los otros e } \\
\text { intenta dominar la relación. }\end{array}$ & & & - & + \\
\hline \multicolumn{5}{|l|}{ 3. REGULACIÓN DE LAS EMOCIONES } \\
\hline 3.1. Regula las emociones. Baja ansiedad. & & & - & - \\
\hline 3.2. Hiperactiva las emociones y/o las exterioriza mediante acting out. Alta ansiedad. & & & + & \\
\hline 3.3. Desactiva las emociones. Baja ansiedad. & & & - & + \\
\hline \multicolumn{5}{|l|}{ 4. REVELACIÓN DE LAS EMOCIONES } \\
\hline 4.1. Autorrevelación adecuada en las relaciones. & & & - & - \\
\hline 4.2. Autorrevelación excesiva y/o indiscriminada. & & & + & - \\
\hline 4.3. Dificultad para autorrevelar. & & & & + \\
\hline Total & & & & \\
\hline
\end{tabular}

'En cada recuadro se anota la frecuencia de la categoría, es decir, el número total de veces en que aparece en la narrativa del paciente, así como el valor de probabilidad. 2 El signo (-) o (+) indica que el valor resta o suma en las dimensiones de ansiedad y evitación. 


\begin{tabular}{|c|c|l|}
\hline Ansiedad & Evitación & Apego \\
\hline- & - & Seguro \\
\hline+ & - & Preocupado \\
\hline+ & + & Temeroso \\
\hline- & + & Evitativo \\
\hline
\end{tabular}

revelación de las emociones (4.1., 4.2., 4.3.). Debe tenerse en cuenta que en una unidad de análisis puede haber información relevante para más de una categoría.

3. Contabilizar la frecuencia total de narrativas clasificadas en cada categoría y convertir las frecuenci- as en valores de probabilidad, calculando el cociente entre la frecuencia de cada categoría y el número total de observaciones. Mediante la conversión en valores de probabilidad se busca poder comparar a pacientes en el tipo de verbalizaciones que realizan sobre sus relaciones, con independencia de que dichas verbalizaciones sean muchas o pocas.

4. Sumar los valores de cada categoría en función de si indican la presencia/ausencia de ansiedad y/o la mayor/menor evitación.

5. Determinar el tipo de apego predominante y su posición gráfica en función de las puntuaciones en ansiedad y evitación. 